\title{
High-redshift Massive Quiescent Galaxies Are as Flat as Star-forming Galaxies: The Flattening of Galaxies and the Correlation with Structural Properties in CANDELS/ 3D-HST
}

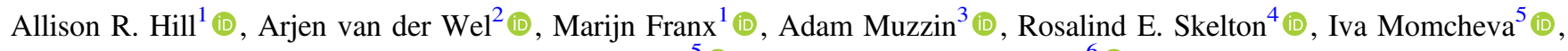 \\ Pieter van Dokkum ${ }^{5}$ (D), and Katherine E. Whitaker ${ }^{6}$ (D) \\ ${ }^{1}$ Leiden Observatory, Leiden University, P.O. Box 9513, 2300 RA, Leiden, The Netherlands; hill@strw.leidenuniv.nl \\ ${ }^{2}$ Department of Physics and Astronomy, Ghent University, 9000 Gent, Belgium \\ ${ }^{3}$ Department of Physics and Astronomy, York University, 4700 Keele St., Toronto, ON MJ3 1P3, Canada \\ ${ }^{4}$ South African Astronomical Observatory, PO Box 9, Observatory, Cape Town, 7935, South Africa \\ ${ }^{5}$ Astronomy Department, Yale University, New Haven, CT 06511, USA \\ ${ }^{6}$ Department of Physics, University of Connecticut, Storrs, CT 06269, USA \\ Received 2018 April 17; revised 2018 November 5; accepted 2018 November 16; published 2019 January 23
}

\begin{abstract}
We investigate the median flattening of galaxies at $0.2<z<4.0$ in all five CANDELS/3D-HST fields via the apparent axis ratio, $q$. We separate the sample into bins of the redshift, stellar mass, Sérsic index, size, and $U V J-$ determined star-forming state to discover the most important drivers of the median $q\left(q_{\text {med }}\right)$. Quiescent galaxies at $z<1$ and $M_{*}>10^{11} M_{\odot}$ are rounder than those at lower masses, consistent with the hypothesis that they have grown significantly through dry merging. The massive quiescent galaxies at higher redshifts become flatter and are as flat as star-forming massive galaxies at $2.5<z<3.5$, consistent with formation through direct transformations or wet mergers. We find that in quiescent galaxies, correlations with $q_{\text {med }}$ and $M_{*}$, and $z$ and $r_{e}$ are driven by the evolution in the Sérsic index $(n)$, consistent with the growing accumulation of minor mergers at lower redshifts. Interestingly, $n$ does not drive these trends fully in star-forming galaxies. Instead, the strongest predictor of $q$ in star-forming galaxies is the effective radius, where larger galaxies are flatter. Our findings suggest that $q_{\text {med }}$ is the tracing bulge-to-total ratio, which would explain why smaller/more massive star-forming galaxies are rounder than their extended/less massive analogs, although it is unclear why the Sérsic index correlates more weakly with flattening for star-forming galaxies than for quiescent galaxies.
\end{abstract}

Key words: galaxies: evolution - galaxies: formation - galaxies: structure

\section{Introduction}

Tracing the morphological evolution of galaxies from photometry is valuable in providing insights into the underlying kinematics of galaxy evolution when time-expensive, high signal-to-noise $(\mathrm{S} / \mathrm{N})$ spectra are unavailable. Physical parameters have long been known to couple broadly to the Hubble type (e.g., Roberts \& Haynes 1994; Blanton et al. 2003), with young, star-forming (SF) galaxies exhibiting some form of gas-rich disk or flattened structure and quiescent $(\mathrm{Q})$ galaxies exhibiting older stellar populations in rounder, puffed up ellipticals (although passive disks do make up a small, but not insignificant, population of passive galaxies; e.g., Bruce et al. 2014a).

In order to quantify the morphological evolution, various structural parameters have proven to be useful proxies for visual classification. In general, disk galaxies have been associated with a low $(n \sim 1)$ Sérsic index surface brightness profile (or an exponential profile), and elliptical galaxies are associated with a high $(n \sim 4)$ Sérsic index light profile (de Vacouleurs profile). Along with a Sérsic parameter, galaxies have also been quantified based on their effective radius, $r_{e}$, and their apparent axis ratio, $q$.

On a galaxy-by-galaxy basis, $q$ is not in itself a very useful parameter as it can depend strongly on the inclination angle. However, distributions of $q$ have been used to infer the intrinsic axis ratios of populations of galaxies separated by their Hubble type (e.g., Sandage et al. 1970; Lambas et al. 1992) and by the mass, star-forming state, and redshift (e.g., Law et al. 2012;
Chang et al. 2013; van der Wel et al. 2014b). For instance, in the local universe, Lambas et al. (1992) found that the elliptical $q$ distribution implied that these galaxies are intrinsically triaxial as pure oblate/prolate models could not account for the observed axis ratio distributions.

van der Wel et al. (2014b) and Chang et al. (2013) used similar methodology to measure how the distributions evolve with redshifts in star-forming and quiescent galaxies. Chang et al. (2013) confirmed that the apparent axis ratio distribution of quiescent galaxies at low- $z$ is consistent with intrinsic triaxial shapes, and that this is also true in their high-redshift $(1<z<2.5)$ counterparts. They also found that at $z>1$, galaxies with $M_{*} \sim 10^{11} M_{\odot}$ exhibited a higher oblate fraction, which they interpreted as massive galaxies being comprised of disks in the past that were destroyed in major-merger events. For lower-mass quiescent galaxies $\left(M_{*}<10^{10.5} M_{\odot}\right)$, the evolution of the oblate fraction is reversed, with low-mass quiescent galaxies at high- $z$ not having sufficient time to settle into stable disk systems as compared to today.

In star-forming galaxies, van der Wel et al. (2014b) found that disks are ubiquitous among massive galaxies at all redshifts below $z \sim 2$. At a lower stellar mass $\left(M_{*}<\right.$ $10^{10} M_{\odot}$ ), the fraction of galaxies with elongated intrinsic shapes increases toward higher redshifts and lower masses, and that similar to their low-mass quiescent counterparts discussed in Chang et al. (2013), these galaxies did not have sufficient time to settle into stable disks. This interpretation is supported by the kinematic analysis in integral field unit (IFU) studies, such as Simons et al. (2017) who find that disordered (i.e., 
dispersion dominated) motions decrease with decreasing redshifts in low-mass star-forming galaxies.

In this study, we chose to investigate the median apparent axis ratio $\left(q_{\mathrm{med}}\right)$ evolution instead of modeling the distributions and inferring their intrinsic shapes. We instead inferred the intrinsic flattening from the median flattening, with the underlying assumption that the trends in the median encapsulate trends in the larger population. We caveat this with the fact that many studies that investigated the apparent axis ratio distribution, $P(q)$, found that a single morphological type often does not reproduce the observed $P(q)$ and that the models demand a more heterogeneous population (e.g., Lambas et al. 1992; Chang et al. 2013; van der Wel et al. 2014b). Using the $q_{\text {med }}$, we can quantify the dependency on other structural parameters, such as $n$ and $r_{e}$ and their evolution. We analyzed how these values change as a function of the star-forming state of these galaxies and determine what $q_{\text {med }}$ is tracing in these different populations.

We noted that the apparent average flattening of a population of galaxies is closely related to the average intrinsic flattening defined by the ratio of the short axis to the long axis of a galaxy (see, e.g., Franx et al. 1991). The ratio of the intermediate axis to the long axis only weakly influences the apparent flattening.

Throughout this paper, we assumed a $\Lambda$-cold dark matter (CDM) cosmology $\left(H_{0}=70 \mathrm{kms}^{-1} \mathrm{Mpc}^{-1}, \Omega_{M}=0.3\right.$, and $\left.\Omega_{\Lambda}=0.7\right)$.

\section{Sample Selection}

This work makes use of the structural parameter catalogs of van der Wel et al. (2012), which were generated using GALFIT (Peng et al. 2010). We used the parameters in the observed F160W band, which corresponds to the $H$ band. These authors constructed point-spread functions (PSFs) in a hybrid way: the outskirts of the PSFs are derived from stacked stars in the image; the area within a radius of 3 pixels is based on theoretical PSFs constructed by TinyTim (Krist 1995) and processed in the same way as the raw science data. GALFIT is used to fit to each individual galaxy. Neighboring objects are masked out if they are substantially fainter than the main target; otherwise, they are included in the fit (see van der Wel et al. 2012 for more details).

We also utilized the most recent (v4.1.5) photometric catalogs on which they are based from the Cosmic Assembly Near-infrared Deep Extragalactic Legacy Survey (CANDELS)/3D-HST) survey (Brammer et al. 2012; Skelton et al. 2014; Momcheva et al. 2016). We used the stellar population parameters and rest-frame colors based on the "zbest" catalogs, which will use (if available) first a spectroscopic redshift, then a (good) grism redshift, and last a photometric redshift if a spectroscopic and grism redshift were not available. Stellar masses were estimated from fits of stellar population models to the full photometric data set (ranging from the UV to $4.5 \mu \mathrm{m}$ ). We refer the reader to the aforementioned papers and their associated documentation for more details. ${ }^{7}$

We performed a first pass selection using the 3D-HST photometric flags (use_phot $=1$ ), as well as an F160W magnitude cut of $m_{A B}=24.5$ to ensure uncertainties in the size and shape were within $10 \%$ (as described in van der Wel et al. 2012). We used objects with a quality flag of $f=0,1$ in van der Wel et al. (2012), which means that GALFIT

\footnotetext{
https://3dhst.research.yale.edu/Data.php
}

converged on a solution (without crashing) and that the solution did not require parameters to take on their "constraint" values.

We also separate our sample into SF and Q galaxies based on their rest-frame $U-V$ and $V-J$ colors, where galaxies display a color bi-modality and separate them based on specific star formation rates (Labbé et al. 2005; Williams et al. 2009, 2010; Whitaker et al. 2011). We used the UVJ boundaries defined in Muzzin et al. (2013) to separate the Q and SF sequences.

In Figure 1, we plotted the $\mathrm{F} 160 \mathrm{~W} \mathrm{AB}$ magnitude and the fraction of "good" structural fits ( $f=0,1$ in van der Wel et al. 2012) as a function of the mass and redshift, as well as the SF state to determine our mass completeness as a result of our magnitude limit and the effect of our decision to take only "good" structural parameters. In the top panels, we indicated the mass completeness limit for each redshift (which ranges from $\log M_{*} / M_{\odot}=9.5-11.0$ ), to ensure a sufficient $\mathrm{S} / \mathrm{N}$. In the bottom panels, we see that the fraction of "good" structural fits using our mass and magnitude selection is always greater in the SF galaxies, likely because of the difference in their restframe optical colors. This is particularly striking for quiescent galaxies at the highest redshift bin $(3.0<z<4.0)$ at $\log M_{*} / M_{\odot}<10.5$ where we see the recovery of "good" fits is $\sim 30 \%$. However, our mass cut from the top panels ensures we have recovered $>80 \%$ of the total galaxies in each redshift bin.

After applying all of the aforementioned selection criteria to the complete 3D-HST catalog, we are left with 9301 galaxies. A census of these galaxies broken down into their respective redshift and $U V J-S F$ state can be found in Table 1.

\section{Analysis \\ 3.1. Correcting for Systematics}

Since we took a median of $P(q)$, and we have already imposed a fairly conservative $\mathrm{S} / \mathrm{N}$ cut, our random errors on the median are a fraction of a percent for most data points in this work. However, the systematics in $q$ can be significant at the faintest magnitudes. Since we wished to investigate the trends with flattening out to significant $z$, rather than exclude these galaxies from our sample, we chose to correct for the systematics investigated by van der Wel et al. (2012).

In their work, van der Wel et al. (2012) used model light profiles convolved with the noise and PSF profiles of the HST to estimate the effects of systematics. They repeated their surface brightness profile fitting on the simulated images and found that near the magnitude limits of their survey, the measured $q$ in the data were flatter than the model images. In their Table 3, they tabulated the average systematic as a function of the F160W magnitude, which we plotted in Figure 2. Notice that van der Wel et al. (2012) list "simulation output-model input" in their Table 3 . Hence the correction values shown in Figure 2 are the opposite of the listed values, as we show the term that is added to the observed data. In the left panel of Figure 2, we fit an exponential function to the data and made corrections to the values of $q$ in the catalog based on each object's F160W magnitude. Although we did not know the magnitude of the systematic for any individual object, our approach with medians means we can apply these corrections. In the right panel of Figure 2, we show the median correction as a function of $z$. As expected, the magnitude of the 

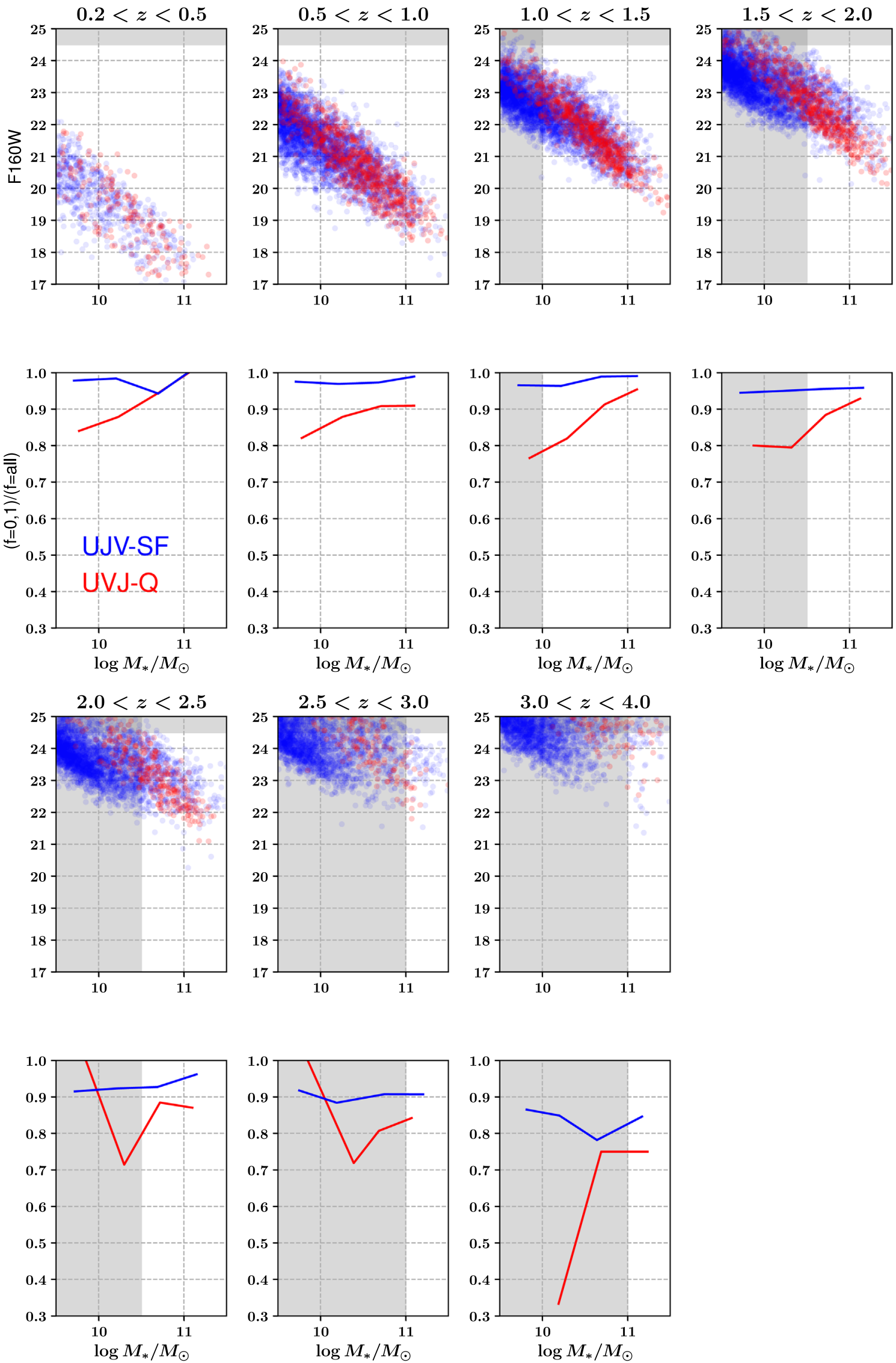

Figure 1. Top panels are the F160W magnitude plotted against the mass, with each panel showing a different redshift bin. The bottom panels show the corresponding recovery of "good" fits (i.e., a flag value of 0 or 1 in the van der Wel et al. 2012 catalogs) as a function of the UVJ star-forming state. The gray-shaded region marks our selected mass and magnitude completeness limits for this study, with the mass limit evolving with increasing $z$. 

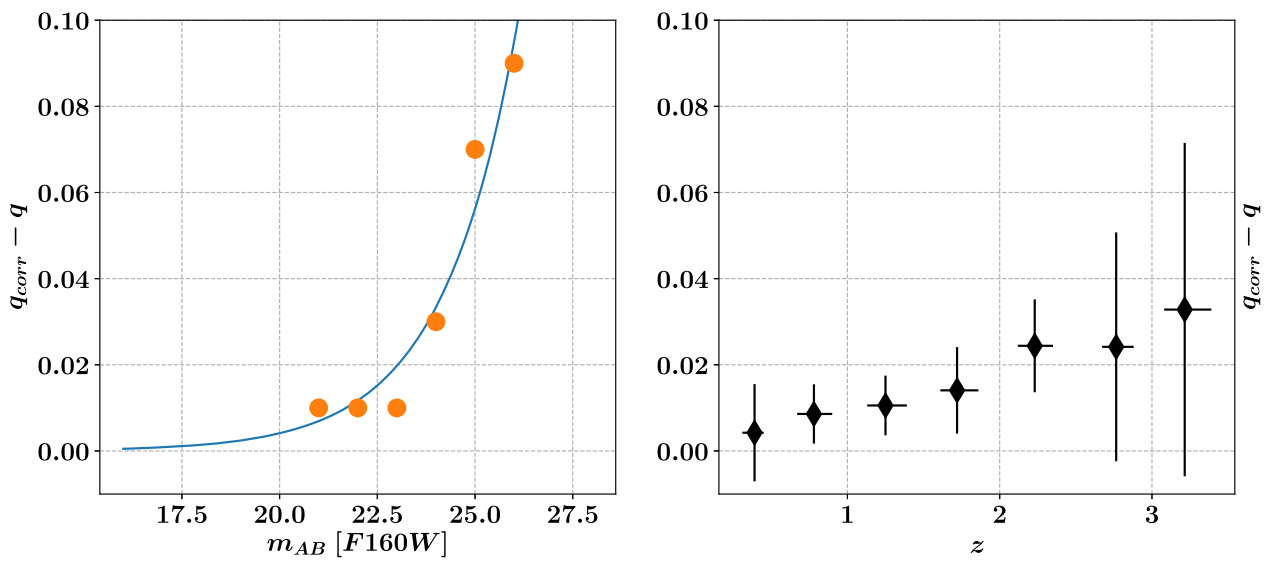

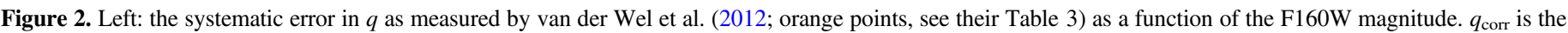

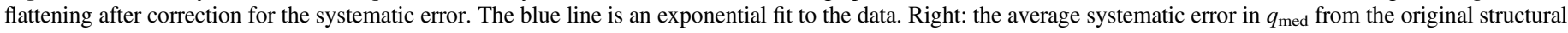

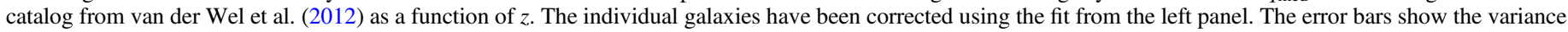
in values. As expected, the total effect of the systematics grows bigger with the redshift.

Table 1

The Number of Galaxies in Each Redshift Range by the UVJ-SF State

\begin{tabular}{lcc}
\hline \hline$z$-range & Quiescent & Star-forming \\
\hline $0.2<z<0.5$ & 173 & 589 \\
$0.5<z<1.0$ & 781 & 3426 \\
$1.0<z<1.5$ & 643 & 1904 \\
$1.5<z<2.0$ & 357 & 614 \\
$2.0<z<2.5$ & 187 & 477 \\
$2.5<z<3.0$ & 16 & 78 \\
$3.0<z<4.0$ & 12 & 44 \\
\hline
\end{tabular}

Note. Above is the number of galaxies in each redshift range that are above our mass limits outlined in Figure 1.

correction is larger at a higher redshift, where the sample is dominated by objects at a fainter magnitude limit (as seen in the top panel of Figure 1).

Unless otherwise specified, the values of $q$ presented in this paper are corrected for these systematic effects.

Another potential systematic can be caused by the shifting intrinsic bandpass as a function of the wavelength. We tested the effect of the bandpass on the axis ratio in two ways. First, we used the analysis of the Galaxy And Mass Assembly (GAMA) survey (Kelvin et al. 2012). These authors derived the flattenings in bands ranging from the $u$ band to the $K$ band. We find that the difference in the median flattening is very small for this sample. When expressed as a function of $\log$ (wavelength), it is $d \log q_{\text {med }} / d \log \lambda=0.00$ for quiescent galaxies and 0.05 for star-forming galaxies. This is measured between the $g$ band and the $H$ band, which is a range representative for our sample. The effect on our results is negligible. In addition, we used the CANDELS photometry itself to estimate the effect by comparing the flattening of the F125W and F160W bands. We found $d \log q_{\text {med }} / d \log \lambda=0.06 \pm 0.03$ and $0.11 \pm 0.024$ for quiescent and star-forming galaxies. The effect for star-forming galaxies somewhat higher than estimated from GAMA, but is consistent at the $2.5 \sigma$ level. It suggests that the dependence of flattening on that passband may depend on the redshift. It would still lead to very small systematics. We tested whether this correction would affect our results; and we only found a small difference for the flattening of the star-forming galaxies as a function of the redshift (Figure 4), where the trend changes by about 0.02 per unit redshift. This is a very small trend that will be ignored in the rest of the analysis.

\subsection{Trends with Star Formation, $M_{*}, z, r_{e}$, and $n$}

To investigate trends in $q_{\text {med }}$ with other properties, we binned our galaxies into seven different redshift bins (with ranges specified in Table 1), as well as four different stellar mass bins $\left(\log M_{*} / M_{\odot} \in[9.5,10.0],[10.0,10.5],[10.5,11.0]\right.$, and $[11.0,12.0])$, three bins of $r_{e}\left(r_{e}[\mathrm{kpc}] \in[0,3],[3,6]\right.$, $[6,9]$, and $[9,20])$, and three bins of $n(n \in[0,2.0]$, [2.0, 4.0], and $[4.0,8.0])$. We excluded galaxies with $r_{e}<0$ ". 1 from our sample, as this is smaller than the HWHM of the PSF. The median $q_{\text {med }}$ are derived for the various bins, and the errors are determined from a bootstrap procedure. Bootstrap resamples are constructed and the medians are determined. The error bars shown in the figures are the rms deviations derived from the distribution of bootstrap medians.

In Figure 3, we plotted $q_{\text {med }}$ as a function of $\log M_{*} / M_{\odot}$ and $z$. In this figure, we only plotted our results to $z=2.5$ because we are not complete in the mass above this redshift (although we plotted our highest mass bin, $M_{*}>10^{11} M_{\odot}$, where we are complete in Figure 4). Considering only the quiescent galaxies, we calculated the average linear least squares slope $\left(\alpha_{\text {avg }}\right)$ for every redshift bin and found $\alpha_{\text {avg }}=0.01 \pm 0.01$, which is consistent with $q_{\text {med }}$ being independent of $M_{*}$. On the other hand, star-forming galaxies at $z<1$ display a broad mass dependence $\left(\alpha_{\mathrm{avg}}=0.05 \pm 0.02\right)$, with lower-mass galaxies appearing flatter than higher mass galaxies. Because we are mass-limited, whether or not this trend continues at $z>1$ is an open question that would require deeper survey depths to answer.

If we now consider the broad difference between quiescent and star-forming galaxies in Figure 3, we see that the quiescent galaxies are generally rounder than their equivalent mass SF counterparts. The exception to this is in our $2.0<z<2.5$ redshift bin, where at $\log M_{*} / M_{\odot}>11.0$, the axis ratios are indistinguishable. This could be indicative of similar morphology between the two populations at these redshifts.

We investigate this similarity to higher redshifts by only considering galaxies in our highest mass bin where we have sufficient redshift coverage given our mass-complete limits. In Figure 4, we plotted the apparent axis ratio of galaxies in our highest mass bin as a function of the redshift. We see that 

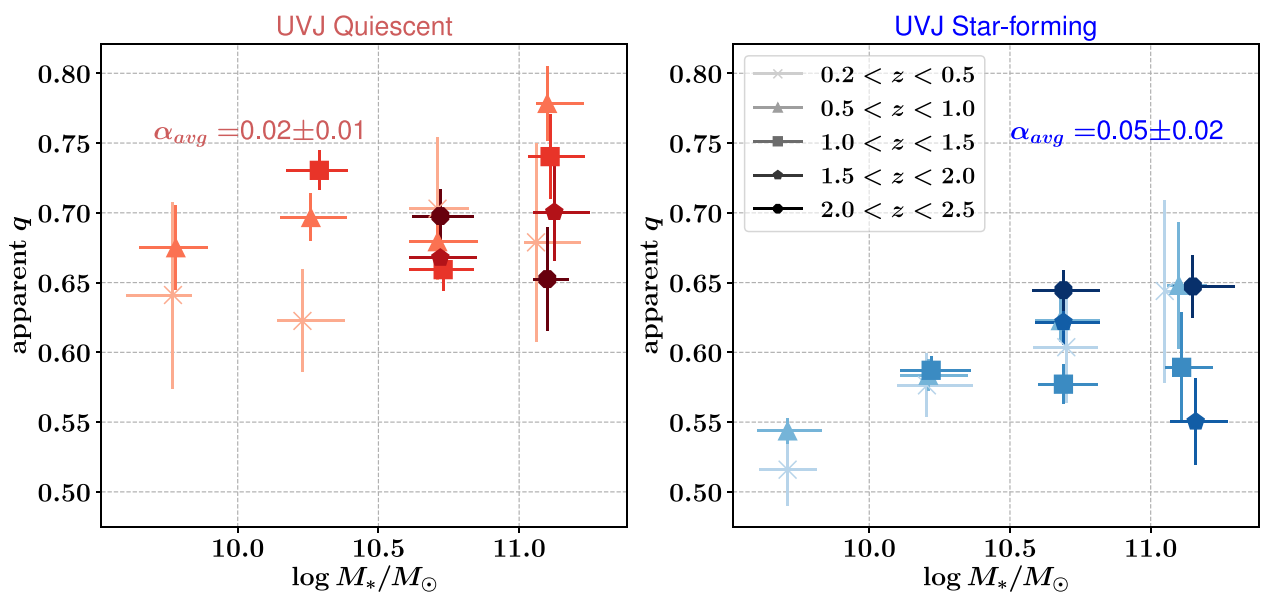

Figure 3. Apparent axis ratio as a function of the mass and redshift for both $U V J$-quiescent (left) and $U V J-\mathrm{SF}$ (right). The quiescent galaxies are rounder than the starforming galaxies; and the quiescent galaxies do not show a strong trend with mass. On the other hand, the star-forming galaxies do show a trend with the mass: the more massive galaxies are rounder. The error bars in $\log M_{*} / M_{\odot}$ represent the interquartile range, and the error bars in $q_{\text {med }}$ are the $1 \sigma$ range from a bootstrapped median and represents the variance. $\alpha_{\text {avg }}$ is the average best-fit slope for $z<1.5$ (i.e., that is the redshift ranges that had at least three data points).
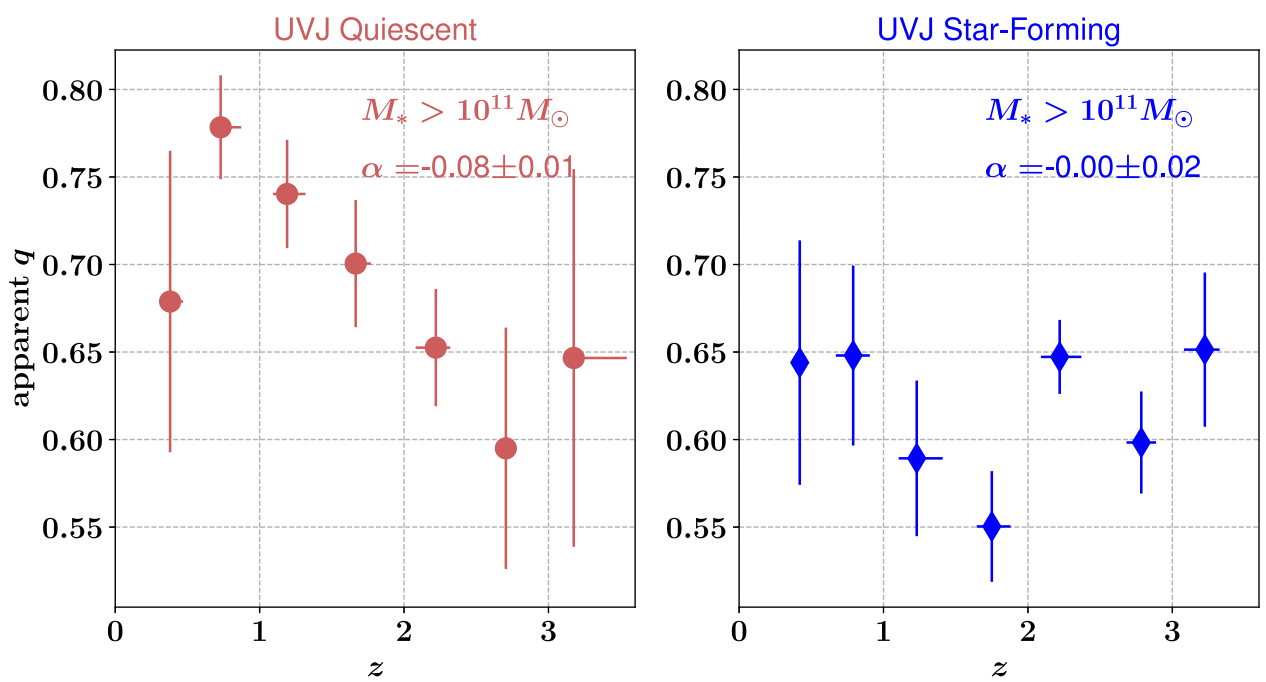

Figure 4. Apparent axis ratio as a function of the redshift, separated into quiescent (left) and star-forming galaxies (right) via a $U V J$ color selection for galaxies at $M_{*}>10^{11} M_{\odot}$. The error bars in $q_{\text {med }}$ are from the bootstrapped median and are representative of the scatter, and the error bars in $z$ show the interquartile range. $\alpha$ is the best-fit, linear least squares slope. Here we see that the quiescent galaxies are rounder than the star-forming galaxies at $z<2$, but are comparable at $z>2$. We also note that the apparent axis ratio has shown significant evolution in quiescent galaxies, but the trend in $q_{\text {med }}$ with $z$ for star-forming galaxies is flat.

quiescent galaxies are flatter at higher redshifts of equivalent masses, whereas the star-forming galaxies show little evolution in $q_{\text {med }}$ with redshifts. As in Figure 3, at $z<2$, the quiescent galaxies are rounder than their star-forming counterparts. At $z>2$, we see that there is no discernible difference in the $q_{\text {med }}$ between the star-forming and quiescent populations, suggesting that at this mass (as alluded to in Figure 3), perhaps these galaxies have a similar structure.

Given the known association between a galaxy's mass and size (e.g., Shen et al. 2003; van der Wel et al. 2014a; Lange et al. 2015) and that the size of galaxies at equivalent masses are observed to be smaller at larger redshifts (e.g., Daddi et al. 2005; Trujillo et al. 2006; Franx et al. 2008; van Dokkum et al. 2008; Straatman et al. 2015), it is also important to determine whether the trends observed in Figure 3 are driven by the size evolution. As previously mentioned, we binned our data according to $r_{e}$ and plotted how this evolves with $z$ and $M_{*}$ in Figures 5 and 6, respectively, but omitted bins with fewer than three galaxies (as has been done for all medians in this work).
In Figure 5, we see that the $q_{\text {med }}$ of star-forming galaxies depends more strongly on $r_{e}$ than their quiescent counterparts (with $\alpha_{\text {avg }}=0.01 \pm 0.004,-0.039 \pm 0.007$ for quiescent and star-forming galaxies, respectively), with large galaxies being flatter than smaller galaxies. At low- $z$, quiescent galaxies become marginally rounder with their increasing size, with this trend disappearing, or even reversing, at $z>2$.

Figure 6 echoes the trends of $r_{e}$ seen in Figure 5 (with starforming galaxies showing steeper $\alpha_{\text {avg }}$ than quiescent galaxies); however, there is a much stronger dependence on $M_{*}$ than with $z$, and massive galaxies are always rounder than less massive galaxies at a fixed $r_{e}$, with the exception of the smallest quiescent galaxies where the trend reverses. These trends are also what are expected if the bulge-to-total galaxy ratio $(\mathrm{B} / \mathrm{T})$ increases with an increasing $M_{*}$ and a decreasing $r_{e}$. In this figure, we also plotted $q_{\mathrm{med}}$ as a function of $r_{e} / r_{e, M_{*}}$, where $r_{e, M_{*}}$ is the expected size given the stellar mass from the mass-size relations of van der Wel et al. (2014a). This can be thought of as a deviation from the mass-size relation. When plotting this fraction instead of the $r_{e}$, we see that the mass 

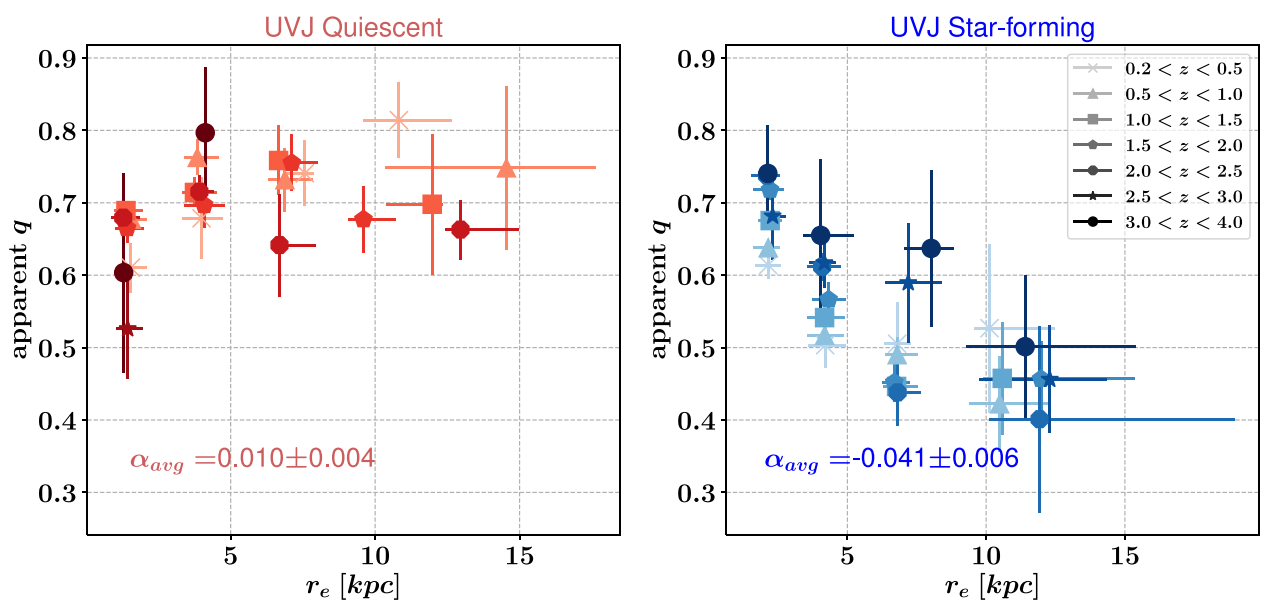

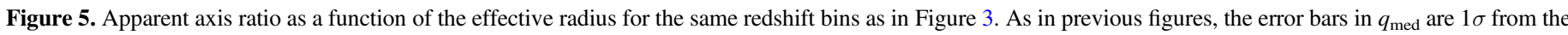

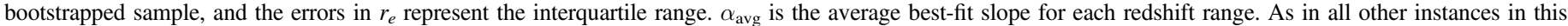

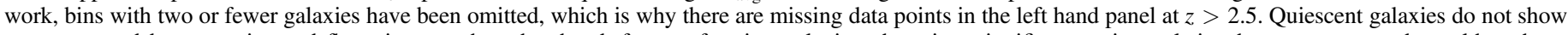

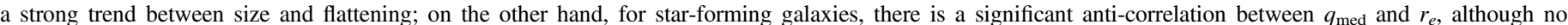
consistent $z$ evolution.

dependence largely disappears in both quiescent and starforming galaxies. In quiescent galaxies, we see a relatively flat relationship. For star-forming galaxies, galaxies that lie below the mass-size relation are rounder than those that lie above it.

In Figure 7, we investigated the dependencies of $n$ on $q_{\text {med }}$ and $M_{*}$. In this figure, the galaxies have been binned by $n$. We observed a strong positive correlation between $q_{\text {med }}$ and $n$ in both quiescent and star-forming galaxies, with no significant $M_{*}$ dependence. Because there is no significant $M_{*}$ dependence, we plotted trend lines in Figure 7 based on the median of all galaxies, as well as only the quiescent/SF in their respective $n$ bins. These lines show that the $n$ dependence is steeper for quiescent galaxies. This is the most significant trend observed out of the structural parameters investigated.

\subsection{Is $n$ Driving Trends with $q_{m e d}$ ?}

Because of the tight relationship between $q_{\text {med }}$ and $n$, we reinvestigate the observed trends with $q_{\text {med }}$ to test the extent to which these trends can be explained by trends in $n$. To this end, we recalculate $q_{\text {med }}$ using their measured values of $n$, as well as the relationships for star-forming and quiescent galaxies in Figure $7\left(q_{n}\right)$. We then take the residual between $q_{\text {med }}$ and $q_{n}$ and plotted that against $M_{*}, z$, and $r_{e}$.

Figure 8 shows the residuals of the values in Figure 3. In this figure, we see, for most data points, that the residuals are $\sim 10 \%$ of the original values and can account for most of the observed $q_{\text {med }}$. For star-forming galaxies, although, there is a structure in the residuals, and $n$ can also account for the trends, especially at the lowest redshifts.

In Figure 9, we show the residuals of the relationship of our massive galaxy subsample $\left(M_{*}>10^{11}\right)$ with $z$. In massive galaxies, we see that $q_{\text {med }}$ can be fully accounted for by $n$, and the trend of massive galaxies becoming rounder at lower redshifts is also gone, with this relationship accounted for by an evolution in the median $n$. We see the flat relationship with SF galaxies is also maintained. Therefore, we conclude that the evolution in $n$ can account for any $q_{\text {med }}$ evolution in massive galaxies.

Although $n$ can convincingly account for most of the observed $q_{\text {med }}$, as well as trends with $M_{*}$ and $z$, it is insufficient to explain the trends in $r_{e}$ for star-forming galaxies. Figures 10 and 11 are the residuals plots of Figures 5 and 6, respectively. For the quiescent galaxies in Figure 6, we do see that the previously seen mass dependence of $q_{\text {med }}$ at a fixed radius is gone (again with the exception of galaxies at the smallest radius). However, the mass dependence for star-forming galaxies persists, as well as the overall trend with $r_{e}$.

\section{Discussion and Conclusions}

In the previous sections, we investigated the dependence of the observed $q_{\text {med }}$ with various structural parameters. At all masses below $z<2$, the median quiescent galaxy is rounder than their star-forming counterpart (Figure 3). For quiescent galaxies, when binned by $M_{*}$, there was no discernible trend with the mass, whereas star-forming galaxies do show a significant mass dependence at low-redshifts $(z<1.0)$. At the highest masses $\left(M_{*}>10^{11}\right)$, quiescent galaxies are increasingly flat at higher $z$, until they match the apparent $q_{\text {med }}$ of starforming galaxies at $z>2$. This suggests that at the highest redshifts, massive quiescent galaxies are structurally similar to their SF counterparts and that high- $z$ quiescent galaxies could be disk-like, a notion that has been posited previously (e.g., van der Wel et al. 2011; Wuyts et al. 2011; Bruce et al. 2012; Buitrago et al. 2013; Chang et al. 2013; Newman et al. 2015; Hill et al. 2017).

This result is also consistent with studies of nearby relic galaxies, which are thought to be "unprocessed" descendants of high-redshift quiescent galaxies (e.g., van den Bosch et al. 2012; Trujillo et al. 2014; Ferré-Mateu et al. 2017; Y1ldırım et al. 2017).

The observed trend of massive galaxies flattening at higher redshifts (Figure 4) can be explained entirely by the dependence of $n$ on $q_{\text {med }}$. This conclusion was drawn through an analysis of the residuals after subtracting the effect of $n$ from $q_{\text {med }}$. To obtain this correction, we binned our sample according to $n$ and $M_{*}$ and found that $n$ correlates strongly with $q_{\text {med }}$ with no apparent stellar mass dependence (Figure 7). Using the linear relationship surmised in Figure 7, we calculated what $q_{\text {med }}$ would be given the modeled $n$ from the catalog of van der Wel et al. (2012) and plotted the residuals. The residuals for $q_{\text {med }}$ with $z$ in massive galaxies were 

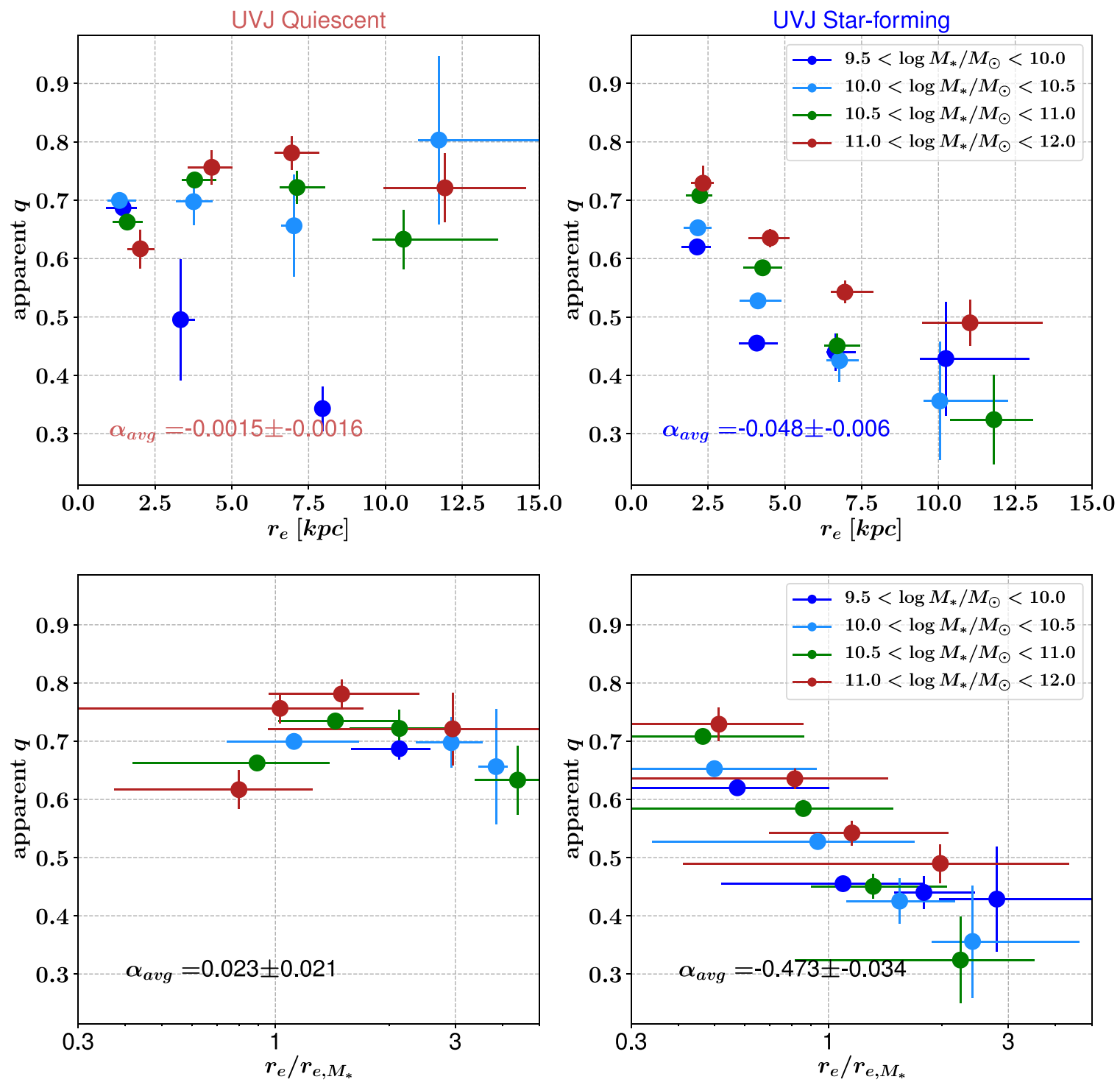

Figure 6. Top: similar to Figure 5, except galaxies have been binned according to $M_{*}$ instead of their redshift. As in other figures, $\alpha_{\text {avg }}$ is the average of the best-fit linear least squares slopes to each mass bin. Bottom: the same as the top row, except instead of plotting the axis ratio against $r_{e}$, we plotted the ratio of $r_{e}$ to the expected size based on its mass from the mass-size relation of van der Wel et al. (2014a; $r_{e, M_{*}}$ ). The differences in the mass bin seen in the top row disappear when considering the deviation from the mass-size relation. We found that the flattening of star-forming galaxies depends strongly on the size normalized to the expected size for the redshift and mass of the galaxies. No strong trend is found for quiescent galaxies.

consistent with 0 (Figure 9), with the conclusion that the evolution in $n$ drives the evolution in $q_{\text {med }}$.

The $q_{\text {med }}$ residuals were also plotted for the other masses, and the residuals were insignificant for the quiescent galaxies. These results are consistent with a simple picture in which quiescent galaxies grow with time due to minor mergers (e.g., van Dokkum et al. 2010), which would make them appear rounder and would increase the Sérsic index. More detailed comparisons with simulations are required to test this explanation in detail.

It is remarkable that the star-forming galaxies show different trends than the quiescent galaxies. This is likely related to the fact that the star-forming galaxies grow through very different mechanisms (e.g., growth through the accretion of gas and subsequent star formation in a disk).

We do not find a strong trend of flattening with the redshift (e.g., Figure 4); on the other hand, the flattening correlates significantly with the mass, very strongly with the effective radius, and with the Sersic index. If we "take out" the correlation with the Sérsic index, we still see a correlation of residual flattening with the effective radius, which is in contrast to the quiescent galaxies.

The most remarkable of these correlations for star-forming galaxies is the correlation with $r_{e}$ : when binning galaxies based on their $r_{e}$, for star-forming galaxies, we observed a negative relationship between $q_{\text {med }}$ and $r_{e}$, with larger galaxies exhibiting stronger flattening than smaller starforming galaxies, regardless of $z$ (Figure 5). This trend persists when comparing star-forming galaxies at a fixed $r_{e}$ in different mass bins (Figure 6). At a fixed $r_{e}$, massive galaxies are always rounder than lower-mass galaxies, regardless of their star-forming state (with the exception of the smallest quiescent galaxies, which requires further investigation). This mass dependence disappears when considering $q$ as a 

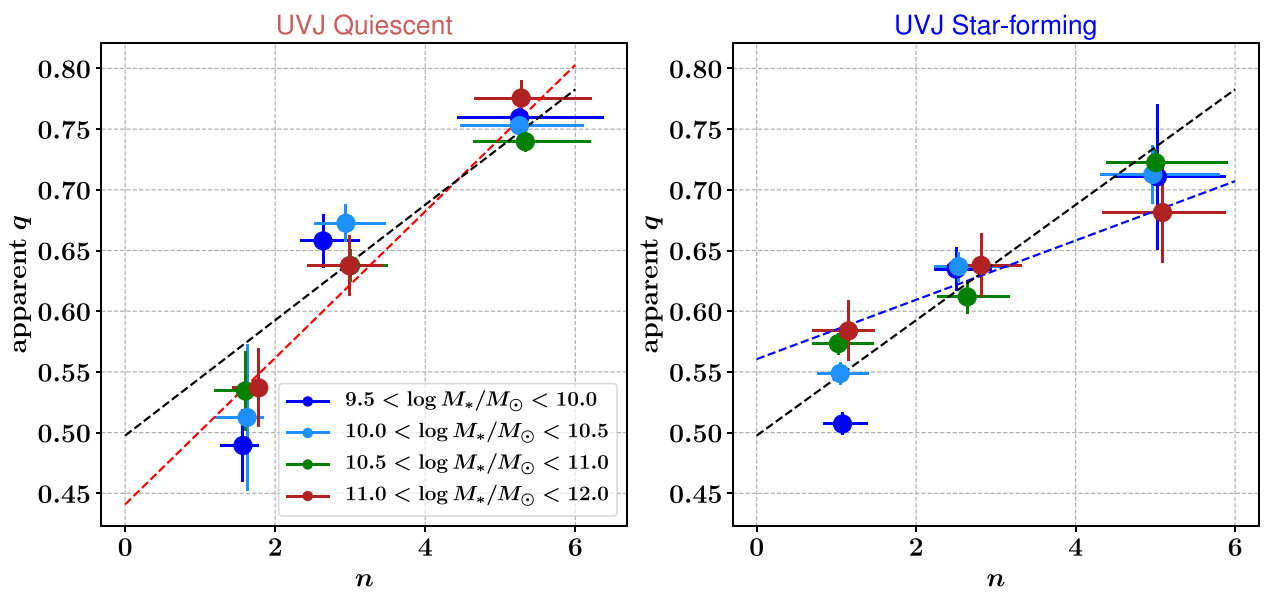

Figure 7. Apparent axis ratio binned by the Sérsic index for four different mass bins for $U V J$-quiescent (left) and UVJ-SF (right). The black dashed lines in both panels are the linear least squares fit to the combined star-forming and quiescent sample. Red and blue dashed lines are the linear fits to the quiescent and star-forming galaxies, respectively. In the left panel, we see no apparent mass dependence in the quiescent galaxies, but we do see a strong correlation of flattening with the Sérsic index. In the right panel, we see a flatter, albeit still strong, relationship between $n$ and $q_{\text {med, }}$, with no apparent mass trend, except in the lowest mass bin where more massive galaxies are rounder. The slopes are $\alpha=0.058,0.062$, and 0.035 for all, quiescent, and star-forming galaxies, respectively.
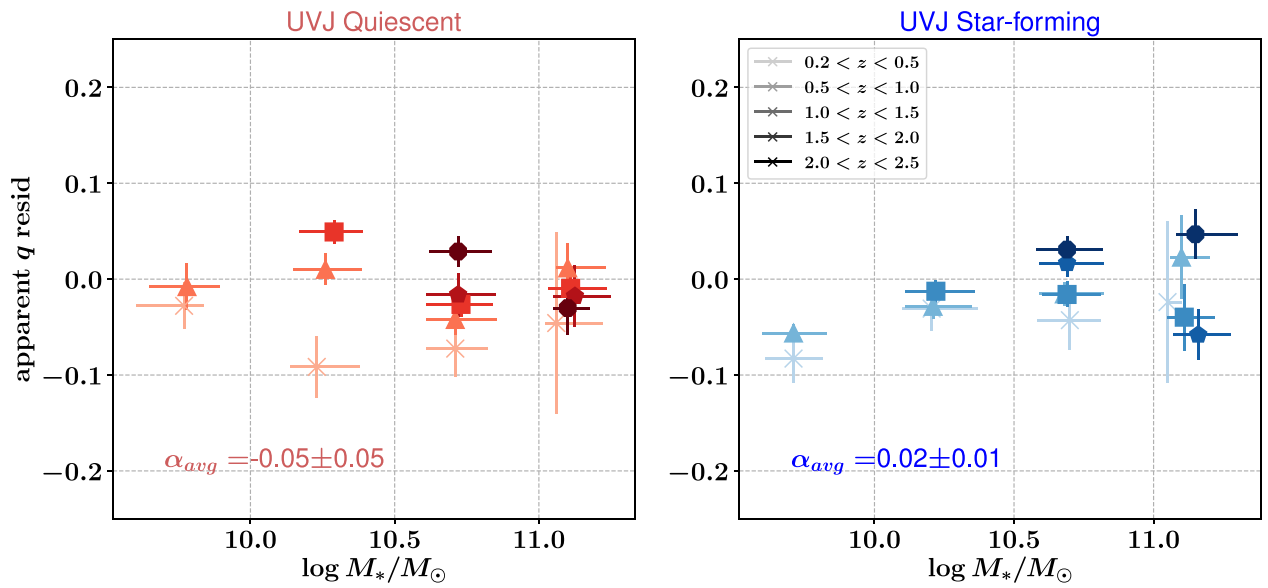

Figure 8. These plots contain the same galaxies and bin as in Figure 3, except the abscissa is now the residual between the actual $q_{\text {med }}$ and $q_{n}$ where $q_{n}$ is the expected $q_{\text {med }}$ based on the galaxies' Sérsic index using the quiescent and star-forming relationships from Figure 7. $n$ is able to account for the observed $q_{\text {med }}$ to within $\sim 10 \%$ for most of the mass and redshift bins. Although there is a structure in the residuals for the star-forming galaxies, the spread in $q_{\text {med }}$ observed in Figure 3 disappears, suggesting that $n$ is sufficient to explain the trends.
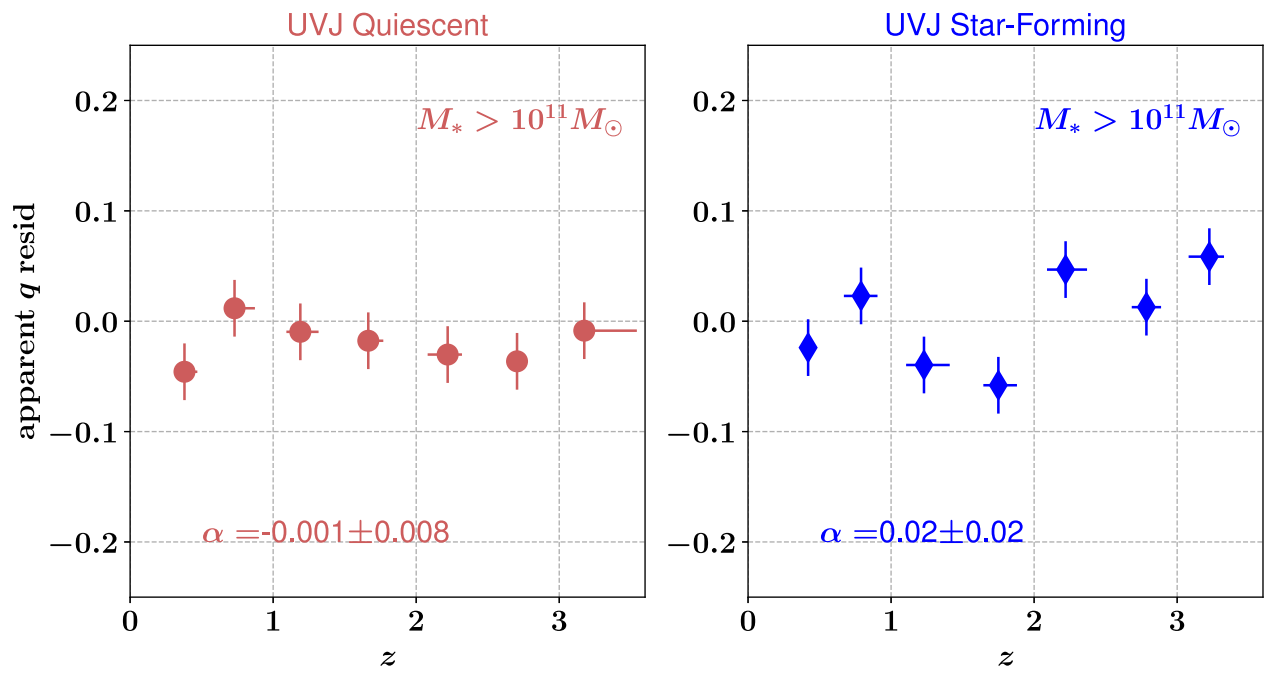

Figure 9. This figure shows the residuals of the relation between flattening and redshifts for the most massive galaxies (Figure 4), after subtracting $q_{n}$ (the expected $q_{\text {med }}$ from a galaxy's $n$, assuming the relationships from Figure 7) for galaxies at $\log M_{*} / M_{\odot}>10^{11}$. The strong trend of $q_{\text {med }}$ with $z$ for quiescent galaxies is reduced to zero, showing that the trend was correlated with a trend in the Sérsic index, $n$, and that $n$ is able to account for the observed $q_{\text {med }}$ for massive galaxies. The trend for the star-forming galaxies is still consistent with zero. 

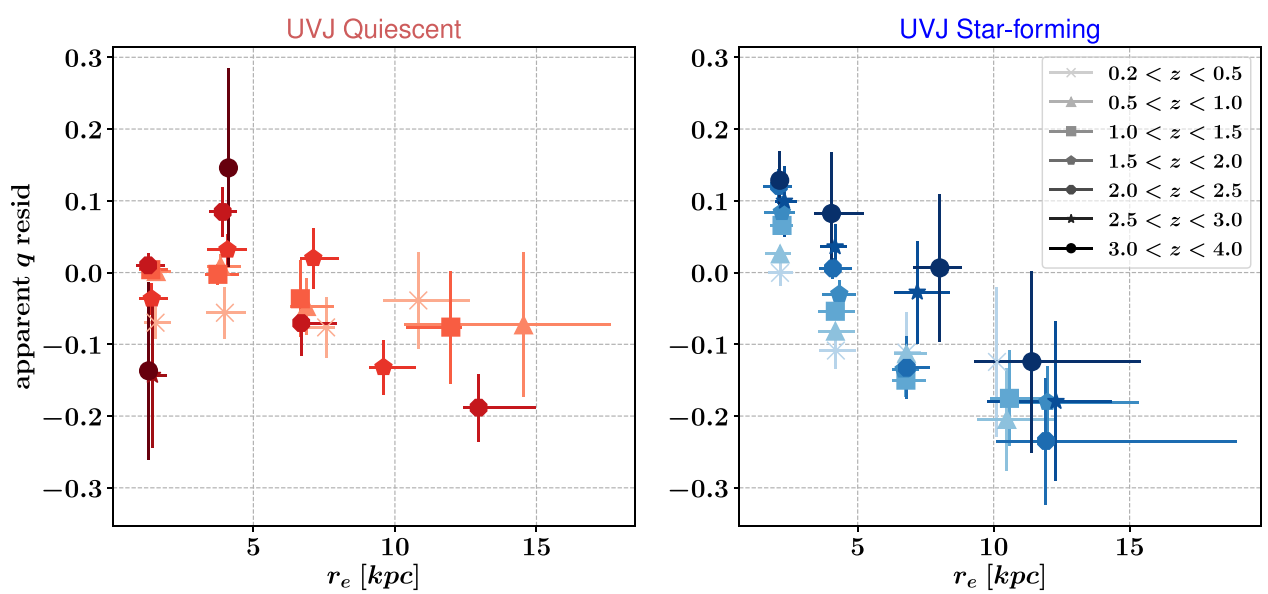

Figure 10. This figure shows the residuals of Figure 5, after subtracting $q_{n}$ (the expected $q_{\text {med }}$ from a galaxy's $n$, assuming the relationships from Figure 7) for galaxies at $\log M_{*} / M_{\odot}>10^{11}$. This trend is sufficient to explain the observed $q_{\text {med }}$ of quiescent galaxies, but does not account for the trend of $q_{\mathrm{med}}$ with $r_{e}$ in star-forming galaxies where the magnitude of the trend persists.
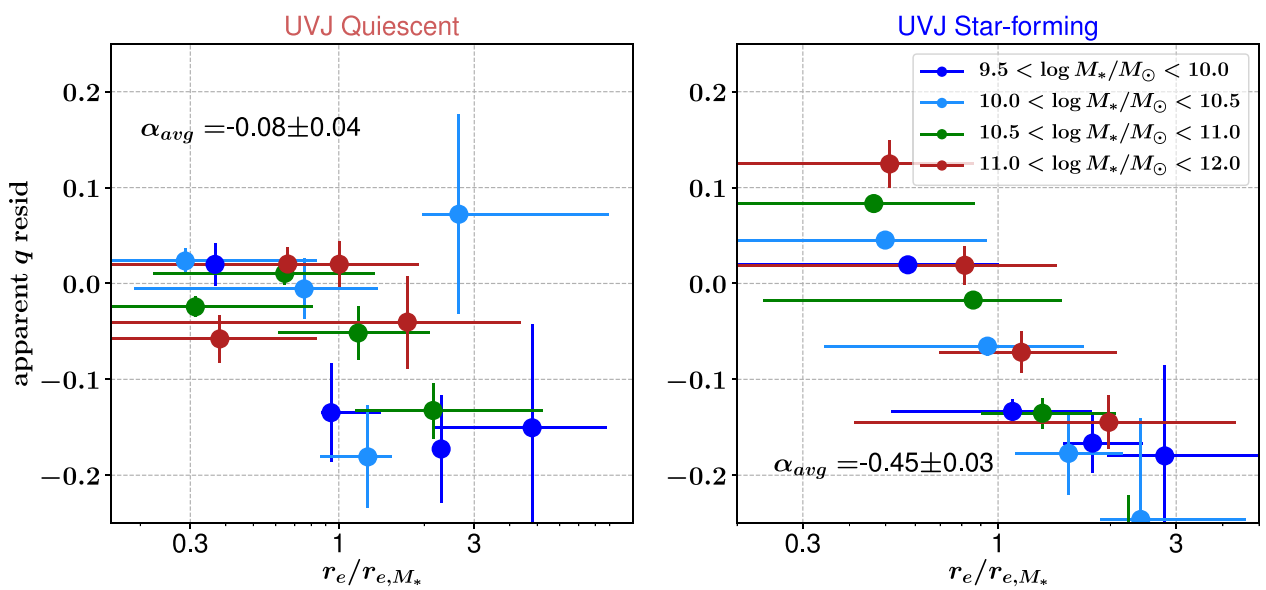

Figure 11. Residual values after subtracting $q_{n}$ (the expected $q_{\text {med }}$ from a galaxy's $n$, assuming the relationships from Figure 7) from $q_{\text {med }}$ in Figure 6. As in Figure 10, $n$ is able to account for $\sim 80 \%-90 \%$ of the observed $q_{\text {med }}$ in quiescent galaxies. In star-forming galaxies, the observed trend with $r_{e}$ does not change after correcting for the correlation with $n$.

function from the deviation of the relevant mass-size relation (Figure 6).

To the first order, the results are interpreted by assuming that $q_{\text {med }}$ is tracing the $\mathrm{B} / \mathrm{T}$ in SF galaxies. It has been shown previously that $n$ broadly traces the $\mathrm{B} / \mathrm{T}$ in massive galaxies (e.g., Bruce et al. 2014b; Kennedy et al. 2016); this, combined with our observation that $q_{\text {med }}$ is also correlated with $n$, makes a consistent picture.

It is not entirely clear, however, why size plays such a dominant role: the flattening varies by a factor of about 2 as a function of the size normalized to the mass-size relationstronger than the variation with the Sérsic index. In addition, when the dependence on the Sérsic index is taken out, there remains a correlation with the size.

Possibly, these effects are simply due to the fact that the light distribution of star-forming galaxies is very sensitive to dust, orientation, and young, unobscured star formation. Hence simple trends for quiescent galaxies become complex. Take, for example, the case of disk galaxies for which the disks almost "disappear" due to dust when viewed edge-on (e.g., Patel et al. 2012). In short, models are needed to interpret these results and to derive the full interpretation.

\section{Summary}

We have taken the catalogs of van der Wel et al. (2012) and studied the evolution of the median apparent axis ratio $\left(q_{\mathrm{med}}\right)$ for over 9000 galaxies out to $z=3$ with $M_{*}, z, n$ and $r_{e}$. We find:

1. Quiescent galaxies are rounder than their SF counterparts at all masses below $z<2$. Above $z>2$, the median flattening between massive quiescent and SF galaxies is identical, suggesting they had a very similar structure in the early universe (Figure 4). This is an extension in the redshift of previous work by Chang et al. (2013), who found an increased incidence of disk-like structures in massive quiescent galaxies at $z>1$.

2. The flattening in quiescent galaxies is mass independent; whereas in star-forming galaxies, there is a steep positive correlation with the stellar mass at least until $z=1$ (Figures 3,4). Due to our mass limits, whether this trend continues to higher $z$ is an open question.

3 . In star-forming galaxies, $q_{\text {med }}$ correlates significantly with $r_{e}$, which is in contrast to quiescent galaxies where there is no discernable trend (Figure 5). 
4. In quiescent galaxies, the strongest common correlation was between $q_{\text {med }}$ and $n$ (Figure 7 ). For most relationships, there is very little residual correlation between $q_{\text {med }}$ and $q_{n}$ (the expected $q$ calculated from the Sérsic index); however, this was not the case in star-forming galaxies (Figure 8).

5. We suspect that $q_{\text {med }}$ is likely tracing the $\mathrm{B} / \mathrm{T}$ ratio, which would explain why smaller/more massive starforming galaxies are rounder than their extended/less massive counterparts, as well as why we do not observe strong $M_{*}$ and $r_{e}$ dependencies in quiescent galaxies, as the majority of the quiescent galaxies are not expected to have prominent disks. We caveat that we are also only tracing the light, which would weight blue disks with lower mass-to-light ratios heavily in the observables and that the mass distribution could be quite different.

We thank the referee for the constructive comments that helped to improve the paper. This research has made use of NASA's Astrophysics Data System. This work is based on observations taken by the 3D-HST Treasury Program (GO 12177 and 12328) with the NASA/ESA HST, which is operated by the Association of Universities for Research in Astronomy, Inc., under NASA contract NAS5-26555. We used the public available programming language PYTHON, including the NUMPY, MATPLOTLIB packages.

\section{ORCID iDs}

Allison R. Hill (1) https://orcid.org/0000-0002-0696-5458 Arjen van der Wel (i) https://orcid.org/0000-0002-5027-0135 Marijn Franx (i) https://orcid.org/0000-0002-8871-3026 Adam Muzzin (iD https://orcid.org/0000-0002-9330-9108 Rosalind E. Skelton (i) https://orcid.org/0000-0001-7393-3336 Iva Momcheva (1D https://orcid.org/0000-0003-1665-2073 Pieter van Dokkum (iD https://orcid.org/0000-0002-8282-9888 Katherine E. Whitaker (i) https://orcid.org/0000-00017160-3632

\section{References}

Blanton, M. R., Hogg, D. W., Bahcall, N. A., et al. 2003, ApJ, 594, 186 Brammer, G. B., van Dokkum, P. G., Franx, M., et al. 2012, ApJS, 200, 13 Bruce, V. A., Dunlop, J. S., Cirasuolo, M., et al. 2012, MNRAS, 427, 1666
Bruce, V. A., Dunlop, J. S., McLure, R. J., et al. 2014a, MNRAS, 444, 1001 Bruce, V. A., Dunlop, J. S., McLure, R. J., et al. 2014b, MNRAS, 444, 1660 Buitrago, F., Trujillo, I., Conselice, C. J., \& Haussler, B. 2013, MNRAS, 428, 1460

Chang, Y.-Y., van der Wel, A., Rix, H.-W., et al. 2013, ApJ, 773, 149

Daddi, E., Renzini, A., Pirzkal, N., et al. 2005, ApJ, 626, 680

Ferré-Mateu, A., Trujillo, I., Martín-Navarro, I., et al. 2017, MNRAS, 467, 1929

Franx, M., Illingworth, G., \& de Zeeuw, T. 1991, ApJ, 383, 112

Franx, M., van Dokkum, P. G., Förster Schreiber, N. M., et al. 2008, ApJ, 688,770

Hill, A. R., Muzzin, A., Franx, M., et al. 2017, ApJ, 837, 147

Kelvin, L. S., Driver, S. P., Robotham, A. S. G., et al. 2012, MNRAS, 421, 1007

Kennedy, R., Bamford, S. P., Häußler, B., et al. 2016, MNRAS, 460, 3458

Krist, J. 1995, in ASP Conf. Ser.77, Astronomical Data Analysis Software and Systems IV, ed. R. A. Shaw, H. E. Payne, \& J. J. E. Hayes (San Francisco, CA: ASP), 349

Labbé, I., Huang, J., Franx, M., et al. 2005, ApJL, 624, L81

Lambas, D. G., Maddox, S. J., \& Loveday, J. 1992, MNRAS, 258, 404

Lange, R., Driver, S. P., Robotham, A. S. G., et al. 2015, MNRAS, 447, 2603

Law, D. R., Steidel, C. C., Shapley, A. E., et al. 2012, ApJ, 745, 85

Momcheva, I. G., Brammer, G. B., van Dokkum, P. G., et al. 2016, ApJS, 225, 27

Muzzin, A., Marchesini, D., Stefanon, M., et al. 2013, ApJ, 777, 18

Newman, A. B., Belli, S., \& Ellis, R. S. 2015, ApJL, 813, L7

Patel, S. G., Holden, B. P., Kelson, D. D., et al. 2012, ApJL, 748, L27

Peng, C. Y., Ho, L. C., Impey, C. D., \& Rix, H.-W. 2010, AJ, 139, 2097

Roberts, M. S., \& Haynes, M. P. 1994, ARA\&A, 32, 115

Sandage, A., Freeman, K. C., \& Stokes, N. R. 1970, ApJ, 160, 831

Shen, S., Mo, H. J., White, S. D. M., et al. 2003, MNRAS, 343, 978

Simons, R. C., Kassin, S. A., Weiner, B. J., et al. 2017, ApJ, 843, 46

Skelton, R. E., Whitaker, K. E., Momcheva, I. G., et al. 2014, ApJS, 214, 24

Straatman, C. M. S., Labbé, I., Spitler, L. R., et al. 2015, ApJL, 808, L29

Trujillo, I., Ferré-Mateu, A., Balcells, M., Vazdekis, A., \& Sánchez-Blázquez, P. 2014, ApJL, 780, L20

Trujillo, I., Förster Schreiber, N. M., Rudnick, G., et al. 2006, ApJ, 650, 18

van den Bosch, R. C. E., Gebhardt, K., Gültekin, K., et al. 2012, Natur, 491, 729

van der Wel, A., Bell, E. F., Häussler, B., et al. 2012, ApJS, 203, 24 van der Wel, A., Chang, Y.-Y., Bell, E. F., et al. 2014b, ApJL, 792, L6

van der Wel, A., Franx, M., van Dokkum, P. G., et al. 2014a, ApJ, 788, 28

van der Wel, A., Rix, H.-W., Wuyts, S., et al. 2011, ApJ, 730, 38

van Dokkum, P. G., Franx, M., Kriek, M., et al. 2008, ApJL, 677, L5

van Dokkum, P. G., Whitaker, K. E., Brammer, G., et al. 2010, ApJ, 709, 1018

Whitaker, K. E., Labbé, I., van Dokkum, P. G., et al. 2011, ApJ, 735, 86

Williams, R. J., Quadri, R. F., Franx, M., et al. 2010, ApJ, 713, 738

Williams, R. J., Quadri, R. F., Franx, M., van Dokkum, P., \& Labbé, I. 2009, ApJ, 691, 1879

Wuyts, S., Förster Schreiber, N. M., van der Wel, A., et al. 2011, ApJ, 742, 96

Yıldırım, A., van den Bosch, R. C. E., van de Ven, G., et al. 2017, MNRAS, 468,4216 